\title{
20. Pädiatrischer Frühling - leider abgesagt!
}

Wir haben uns entschlossen, Ihnen dennoch das im Februar 2020 aufgenommene Gespräch mit emer. Univ.-Prof. Dr. Wilhelm Müller, Kongressleiter des Pädiatrischen Frühlings, zu präsentieren

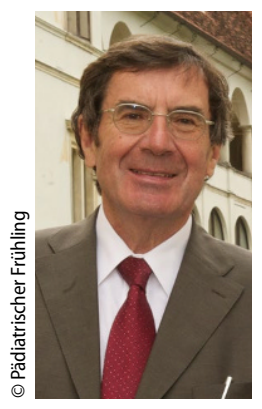

\section{COVID-19 \\ Absage des Pädiatrischen Frühlings 2020}

Schweren Herzens müssen wir den (bereits ausgebuchten) „Pädiatrischen Frühling“ für heuer absagen. Wir beabsichtigen, das für heuer geplante Programm ein Jahr später zu realisieren.

Der Pädiatrische Frühling 2021 ist für 27.-29.05.2021 geplant.

Die Anmeldung dazu wird voraussichtlich ab 15.01.2021 freigeschaltet sein.

Aktuelle Informationen finden Sie auf der Tagungshomepage

www.pädiatrischer-frühling.at

Wir freuen uns schon darauf, Sie 2021 bei hoffentlich bester Gesundheit wieder in

Seggau begrüßen zu dürfen.

Bis dahin alles Gute!

Das Organisationsteam

des Pädiatrischen Frühlings

Pädiatrie \& Pädologie: Der Pädiatrische Frühling wurde erstmals 2001 an der Grazer Kinderklinik abgehalten; wir gratulieren Ihnen herzlich zum 20-jährigen Jubiläum! Was hat sich in diesen 20 Jahren in der Pädiatrie abgespielt?

Müller: Herzlichen Dank für die Gratulation, die ich gerne auch v.a. an Bernhard Resch weitergeben möchte, der gemeinsam mit mir vor 20 Jahren den Pädiatrischen Frühling „erfunden" hat. Und natürlich auch an das gesamte großartige Team!

Ja, wir haben im Jahr 2000 an der Grazer Kinderklinik damit begonnen. Der Hörsaal dort ist dann allerdings sehr schnell zu klein geworden, sodass wir seit 2003 eine (für diesen Zweck ideale!) „Bleibe“ in Schloss Seggau gefunden haben.

Das Format der Tagung wurde in all den Jahren weitgehend beibehalten, die Inhalte haben sich natürlich gewandelt. In den letzten 20 Jahren hat sich fachlich ja enorm viel getan, und darauf wird bei der heurigen Tagung auch Bezug genommen. Organisatorisch sind wir dem Konzept aus Workshops, Plenarvorträgen und Lunchsymposien weitgehend treu geblieben, weil es sich so einfach bewährt hat!

Haben Kinderärztinnen und -ärzte einen besonderen Fortbildungsbedarf?

Ärztinnen und Ärzte sind die einzige Berufsgruppe mit einer Fortbildungsverpflichtung. Aus meiner Sicht besteht allerdings nicht nur eine Fortbildungsverpflichtung, sondern auch eine Fortbildungsnotwendigkeit. Immerhin verdoppelt sich das medizinische Wissen alle drei bis vier Jahre, sodass Fortbildung (auch beruflich) „überlebensnotwendig" ist. Dies trifft ganz besonders für die enorm vielseitige Pädiatrie zu. Denn auch wenn unser Fach leider oft als, „kleines Fach“ tituliert wird, ist es in Wirklichkeit wahrscheinlich das GRÖSSTE klinische Fach! Unsere medizinische Verantwortung reicht immerhin vom 400-Gramm-Frühgeborenen bis zum krankhaft übergewichtigen oder psychosomatisch kranken Jugendlichen. Die Pädiatrie umfasst somit ein riesiges Spektrum von Erkrankungen. Und hier die kompetente Übersicht zu bewahren, gelingt eben nur durch regelmäßige Fortbildung.

Sie verzichten, wie wir wissen, bei dieser Tagung wegen ihrer thematischen Breite seit Anbeginn auf ein Generalthema. Gibt es vielleicht wegen des Jubiläums doch ein Motto?

Es ist vor allem die oben genannte Vielfalt der Pädiatrie, die uns dazu veranlasst, auf ein Schwerpunktthema zu verzichten. Wir arbeiten eigentlich das ganze Jahr über daran, aktuelle Themen zu selektieren und die dazugehörigen Referentinnen und Referenten für die Tagung zu gewinnen. Wobei wir uns bemühen, die „Besten der Besten“ zu bekommen. Davon sind wir auch im Jubiläumsjahr nicht abgegangen.

Allerdings haben wir für heuer unsere Vortragenden gebeten, auch auf die Entwicklung der letzten 20 Jahre einzugehen. Und wenn man diese Vergleiche zwischen 2000 und 2020 anstellt, sieht man auch WIE ENORM die Fortschritte in der Pädiatrie in dieser Zeit waren.

Wie ist das diesjährige Programm zusammengestellt, was erwartet die Teilnehmer?

Am Donnerstag finden wie jedes Jahr Workshops in Kleingruppen statt. Diese sind zwei Wochen nach Öffnung der Anmeldung jetzt schon fast ausgebucht. Am Freitag und Samstag finden dann Plenar- 
vorträge statt, heuer mit folgenden Themenblöcken:

- Infektiologie

- ADHS/Neues vom Sehen

- Reisemedizin

- Neuropädiatrie/Rehabilitation

- Genetik/Seltene Erkrankungen

- Interaktive Fallpräsentationen

Vervollständigt wird das Programm dann durch mehrere Lunchsymposien.

Gibt es Themen, die Sie besonders hervorheben möchten?

An sich erhoffen wir uns, dass jeder einzelne Beitrag ein kleines „Highlight" wird. Wenn ich aber einzelne Themen besonders hervorheben soll, sind das vielleicht die "Reisemedizin" am Freitag und der Themenblock "Genetik/Seltene Erkrankungen" am Samstag. Die "Reisemedizin" wegen der (nicht nur wegen des Coronavirus bestehenden) besonderen Aktualität, und für den Block "Genetik/Seltene Erkrankungen" erwarten wir uns eine für alle gut verständliche und mitreißende Darstellung der modernen Möglichkeiten der Genetik (u.a. durch den „deutschen Dr. House" Herrn Professor Schäfer).

\section{Wie viele Teilnehmer erwarten Sie?}

Wir rechnen damit, dass die Veranstaltung wieder ausgebucht sein wird - insgesamt werden also wieder über 300 Besucher beim Pädiatrischen Frühling erwartet.

\section{Hat die Jubiläumstagung besondere,} feierliche Programmpunkte?

Die sehr strengen ComplianceBestimmungen erlauben ja an sich keine „Belustigungen" mehr. Auf ein (klassisches) Feuerwerk werden wir daher - und auch aus Umweltschutzgründen - verzichten. Wir setzen stattdessen auf ein „Feuerwerk der Vorträge“. Es ist ja letztlich v.a. die Qualität des wissenschaftlichen Programms, das die Kolleginnen und Kollegen nach Seggau bringt.

Aber im Rahmen des Erlaubten werden wir uns natürlich bemühen, dass auch die soziale Komponente nicht zu kurz kommt. Gemeinsames Abendessen, Sport in der Freizeit, und gemeinsames Musizieren sollen die Kommunikation untereinander fördern. An dieser Stelle möchte ich mich auch bei Reinhold Kerbl bedanken, der die Tagung neben der Programmgestaltung immer wieder auch mit neuen Ideen bereichert.

Wann und wo findet der "21. Pädiatrische Frühling" statt?

Auch der 21. Pädiatrische Frühling wird konsequenterweise in Seggau stattfinden. Auch den Termin haben wir schon festgelegt, nämlich für 27.-29.05.2021. Und wir haben auch schon begonnen, das Programm dafür zu erstellen.

Was wünschen Sie sich für die heurige Tagung?

Wir hoffen, dass die Kolleginnen und Kollegen nach drei Tagen heimfahren mit dem Gefühl, viel Neues erfahren bzw. gelernt zu haben, v.a. solche Dinge, die sie in ihrer täglichen Arbeit unterstützen. Und dann wünschen wir uns natürlich auch, dass die Besucher der Tagung auch ausreichend soziale Kontakte pflegen können und uns in ihren Feedbackformularen dann mitteilen:

„Seggau war die Reise wert!"

Wir danken für das interessante Gespräch und wünschen Ihnen und Ihrem Team weiterhin viel Erfolg!

Hinweis des Verlags. Der Verlag bleibt in Hinblick auf geografische Zuordnungen und Gebietsbezeichnungen in veröffentlichten Karten und Institutsadressen neutral.

Paediatr. Paedolog. 2020 · 55:48-49 https://doi.org/10.1007/s00608020-00763-8

(c) Springer-Verlag GmbH Austria, ein Teil von Springer Nature 2020
Das Interview führte Dr. Renate LesskyHöhl, 24.02.2020

Hier steht eine Anzeige.

黛 Springer 